\title{
Quality of Life in Parkinson's Disease Caregivers: The Contribution of Personality Traits
}

\author{
Eloise H. Tew, Sharon L. Naismith, Marilia Pereira, and Simon J. G. Lewis \\ Parkinson's Disease Research Clinic, Brain and Mind Research Institute, University of Sydney, Sydney, NSW 2050, Australia \\ Correspondence should be addressed to Simon J. G. Lewis; simonl@med.usyd.edu.au
}

Received 10 April 2013; Accepted 19 July 2013

Academic Editor: Alfredo Conti

Copyright ( 2013 Eloise H. Tew et al. This is an open access article distributed under the Creative Commons Attribution License, which permits unrestricted use, distribution, and reproduction in any medium, provided the original work is properly cited.

\begin{abstract}
Parkinson's disease imposes significant demands not only on patients but also on those people living and caring for them, who often have a reduction in their quality of life. The factors that may ameliorate these effects, such as an individual's personality, are not understood. Therefore, the aim of this study was to look at the relative contribution of caregiver personality on their quality of life, specifically attempting to identify those traits, which may be protective or harmful. Two hundred and seventy-four caregivers of patients with Parkinson's disease were included in this study. Caregivers were given questionnaires to complete, including the Big Five Inventory and the World Health Organisation Quality of Life BREF version. Univariate correlations demonstrated that depression and anxiety were the largest predictors of reduced quality of life amongst caregivers. However, after controlling for these potential confounds, conscientiousness was associated with enhanced psychological quality of life and openness positively predicted benefits in the environmental domain. Neuroticism was associated with reduced quality of life in the psychological domain. Thus, screening for neuroticism may help identify those caregivers who would benefit from intervention strategies, which could in the long term help reduce the need for nursing home placement of Parkinson's disease patients.
\end{abstract}

\section{Introduction}

Parkinson's disease (PD) is the world's second most common neurodegenerative disease affecting $1 \%-2 \%$ of the population over 65 years of age [1]. It is a progressive condition with both motor and nonmotor symptoms that can have a profound impact not only on the patients but also on family members who often adopt the role of a caregiver [1].

PD can place significant demands on the caregiver, as they take on more daily tasks and increasingly provide physical, emotional, and economic support $[2,3]$. It is recognised that caregivers of people with PD have a reduced quality of life (QoL) [4], where their social activities and work schedules reduce to be more involved in caring [5]. Caregiver spouses are less likely to spend time outside of the house or take a holiday than non-caregiver spouses [5], and older spousal caregivers often have to face age-related challenges themselves.

Preceding data has shown a relationship between advancing stages of PD and a caregiver's QoL, including an increase in strain and a negative lifestyle change as PD progresses [4].
This reduction in QoL can be related to a range of physical, psychological, and sociodemographic factors including the patient's age, disease stage and duration, cognitive and behavioural disorders associated with PD, years of caring, and level of education [2].

Caregiver burden (CB) is a factor that increases in caregivers looking after relatives with PD. CB refers to the stress and impact of looking after a relative and refers to the physical, mental, and socioeconomic problems that caregivers may encounter [6]. There is a significant correlation between increasing $\mathrm{CB}$ and a reduction in a caregiver's QoL [7] and level of depression [8]. Research has shown that PD caregivers have more psychological distress than the general population [9]. This commonly manifests with higher levels of depression, anxiety, and stress, which are associated with increasing disease progression [9].

Whilst a number of factors clearly impact on a PD caregiver's QoL, no known research has examined whether a caregiver's personality confers vulnerability to decreased QoL or conversely offers protection. Personality is an important consideration as it allows for a broader understanding of 
the caregiver's psychological profile. Whilst multiple personality traits have been proposed, there appear to be five key traits, namely extroversion, agreeableness, conscientiousness, openness, and neuroticism [10]. For further review, see Carver and Connor-Smith 2010 [11]. Extroversion describes someone who is confident, has lots of energy, and enjoys social interaction. Agreeableness refers to someone who is friendly, compassionate, and trusting. Conscientiousness refers to when a person is efficient, is organised, shows selfdiscipline, and aims for achievement. Openness, or being open to experience, is used to describe a person who is inventive, curious, and open emotionally. Finally, neuroticism defines someone who is sensitive and nervous and delivers unpleasant emotions easily.

Data has shown that personality characteristics appear to affect the processes people use to appraise stressful events and influence their ability to cope in the absence of caregivers [11]. Furthermore, personality factors relate significantly to QoL [12]. In particular, a number of studies have demonstrated the link between high levels of neuroticism and subsequent stress, decreased QoL, poorer health, and depression in non-caregivers [13-15]. It has been suggested that people expressing neuroticism need better coping methods and relaxation strategies, in order to decrease their psychological distress [11]. In contrast, a high level of extroversion in caregivers of patients with dementia appears to be protective, with data showing it is associated with decreased $\mathrm{CB}$ and depression, which may contribute to an improved QoL [16]. One recent study investigating familial caregivers of community-dwelling older adults with multiple functional impairments demonstrated that whilst extroversion was positively associated with better physical and mental health, neuroticism showed a negative relationship [17]. In addition, conscientiousness, openness, and agreeableness have all been positively associated with better QoL in studies of non-PD caregivers [14].

It has been recognised that caregivers play a pivotal role in preventing the transition of $\mathrm{PD}$ patients to nursing home care [18]. Therefore, identifying PD caregivers at highest risk of reduced QoL is of clinical relevance given that a number of factors, such as the frequency of caregiver breaks and perceived social support, can help to ameliorate the unfavourable effects of caregiving [19]. Such factors are also linked to psychological distress. Therefore, in the current study, we sought to identify the relative contribution of personality traits to various aspects of a PD caregiver's QoL, in the context of other influential factors such as mood.

\section{Materials and Methods}

2.1. Participants. This study assessed 274 caregivers of patients with PD. Caregivers were recruited from both rural $(n=100,36.5 \%)$ and metropolitan areas $(n=174,63.5 \%)$ across New South Wales, Australia. Caregivers included anyone who lived with a participant with $\mathrm{PD}$, whether they were a spouse, relative, or friend.

All caregivers were asked to complete questions that were self-reported, which included demographic questions and psychological questionnaires. Patients were assessed by health professionals with specialist expertise in PD. All procedures were carried out with adequate understanding and written consent in all participants with ethical approval from the University of Sydney.

\subsection{Caregiver Measures}

2.2.1. Personality. The Big Five Inventory-10 (BFI-10) [20] was used to evaluate the five types of personality. BFI-10 was chosen over the BFI-44 because of time constraints and length of the questionnaire pack given to the caregivers. The questionnaire consists of ten questions where two questions relate to each personality domain. The BFI-10 is scored from "Disagree Strongly" (1) to "Agree Strongly" (5) with the highest score indicating more of that personality trait (score range for each domain $=2-10$ ). Some of the five personality factors are measured using a reversed scoring system.

2.2.2. Quality of Life. The World Health Organisation Quality of Life Scale BREF version (WHOQoL-BREF) [21] contains 26 questions measuring QoL using a 5-point scale ranging from "Very Dissatisfied" to "Very Satisfied." There are four domains that can be calculated from the questionnaire; "Physical Health," "Psychological Health," "Social Relationships," and "Environment". The transformed scores from the raw data are ranged from 0 to 100 , with the higher scores indicating a better QoL. The internal consistency, measured by Cronbach's $\alpha$, is satisfactory to good $(\alpha>0.70)$ [22]. Where $>20 \%$ of data was absent from a caregiver's questionnaire, that subject's data was considered missing. Where $>2$ items were missing from a domain, that domain score was not calculated (exception of the social relationship domain, where it was calculated if $<1$ item is missing) [21].

2.2.3. Anxiety and Depression. Psychological distress was measured using the Hospital Anxiety and Depression Scale (HADS) [23], which contains 14 items grouped into two domains: anxiety and depression. The questionnaire is rated on a 4-point response from 0 to 3 with " 0 " indicating no psychological distress at all and " 3 " indicating worse psychological distress. Anxiety and depression were measured individually. Where $>5 \%$ of answers were absent from a domain, scores were considered missing for that domain.

2.2.4. Demographics. Caregiver demographics included age, gender, marital status, relationship with PD patient (spouse, partner, child, or sibling), employment (employed, unemployed, retired/pensioner, or caregiver), education (Pre year 10 , secondary school certificate, technical and further education (TAFE), and university), domestic services required, and any significant caregiver medical and/or mental health concerns. Demographics also included questions about caring for their companion with PD: duration of care (years), whether they received payment for care, what time of the day they help with caregiving (day, night, or both), and the number of hours per day they assist with activities of daily living (ADLs) such as showering, feeding, toileting, assistance with ambulation or transferring, dressing, and grooming. 
2.3. Patient Measures. The physical stage of PD was measured using the Hoehn and Yahr rating scale (H\&Y) [24] and other details including age, gender, marital status, and disease duration (years since diagnosis) were recorded.

2.4. Statistical Analysis. All statistics were generated using the Statistical Package for the Social Sciences (SPSS) version 20.0. Univariate correlations were performed for continuous variables evaluating the relationship between the QoL domains, personality traits, and other contributing factors using Pearson's correlation. Four stepwise linear multiple regressions were then constructed based on significant univariate correlations, with forced entry of key potential confounds in block 1 and personality variables entered into block 2 (method $=$ enter) and the final full model with standardised beta (b) weights and adjusted $R^{2}$ reported. Analyses were two tailed, with an alpha of 0.05 . Correlation strengths were rated as weak (0.2-0.39), moderate (0.4-0.59), and strong $(>0.6)$.

\section{Results}

As shown in Table 1, 65\% of caregivers were females with their spouse/partner as their significant other with PD. The mean age of caregivers was 65 years old and they had been caring for the patient for around four years. Just over half were retired and $29.6 \%$ of caregivers continued their education at University. Participants with PD were slightly older (69.9 years \pm 9.6$)$ and had a disease duration of $7.9( \pm 6.2)$ years with an average $H \& Y$ score of 2.5 .

3.1. Univariate Relationships between QoL and Personality. As shown in Table 2, in the physical health domain, lower levels of caregiver QoL were correlated with greater patient disease duration, advanced stage of disease, older age of the caregiver, greater duration of care, more hours spent assisting with ADLs, higher caregiver neuroticism, anxiety, and depression. Conversely, better physical QoL in caregivers was correlated with higher levels of conscientiousness and openness.

Lower levels of caregiver psychological QoL were associated with greater patient disease duration, advanced stage of disease, longer duration of caring, more hours spent assisting with ADLs, and higher levels of caregiver neuroticism, anxiety, and depression. In contrast, better psychological QoL amongst caregivers was associated with higher levels of conscientiousness and extroversion.

In the social relationship domain, reduced caregiver QoL was associated with advanced stage of disease, longer duration of caring, more hours spent assisting with ADLs, higher levels of caregiver neuroticism, anxiety, and depression. In addition, higher social relationship QoL was associated with higher levels of caregiver conscientiousness.

Lower levels of the environmental QoL were associated with advanced stage of disease, longer duration of caring, more hours spent assisting with ADLs, higher levels of caregiver neuroticism, anxiety, and depression. On the other hand, a better environment QoL score for the caregiver was associated with greater levels of openness.

There were no significant associations between any QoL domain and the personality trait of agreeableness.
TABLE 1: Carer and patient demographic data.

\begin{tabular}{|c|c|c|c|c|}
\hline \multirow{2}{*}{ Demographics } & \multicolumn{2}{|c|}{ Caregiver } & \multicolumn{2}{|c|}{ Patients with PD } \\
\hline & $n$ & $\%$ & $n$ & $\%$ \\
\hline \multicolumn{5}{|l|}{ Region } \\
\hline Metropolitan & 174 & 63.5 & 174 & 63.5 \\
\hline Rural & 100 & 36.5 & 100 & 36.5 \\
\hline \multicolumn{5}{|l|}{ Gender } \\
\hline Female & 177 & 64.6 & 103 & 37.6 \\
\hline Male & 84 & 30.7 & 171 & 62.4 \\
\hline \multicolumn{5}{|l|}{ Marital status } \\
\hline Never married & 11 & 4.0 & 4 & 1.5 \\
\hline Married/de facto & 242 & 88.3 & 241 & 88.0 \\
\hline Separated & 8 & 2.9 & 4 & 1.5 \\
\hline Widowed & 0 & 0.0 & 16 & 5.8 \\
\hline Divorced & 0 & 0.0 & 9 & 3.3 \\
\hline \multicolumn{5}{|l|}{ Relationship } \\
\hline Spouse/partner & 227 & 82.8 & & \\
\hline Child & 25 & 9.1 & & \\
\hline Sibling & 3 & 1.1 & & \\
\hline Friend & 2 & 0.7 & & \\
\hline Other & 4 & 1.5 & & \\
\hline \multicolumn{5}{|l|}{ Employment } \\
\hline Employed & 72 & 26.3 & & \\
\hline Unemployed & 2 & 0.7 & & \\
\hline Retired/pensioner & 152 & 55.5 & & \\
\hline Carer & 24 & 8.8 & & \\
\hline Other & 10 & 3.6 & & \\
\hline \multicolumn{5}{|l|}{ Education } \\
\hline No formal education & 2 & 0.7 & & \\
\hline Preyear 10 & 53 & 19.3 & & \\
\hline Secondary school certificate & 59 & 21.5 & & \\
\hline TAFE & 60 & 21.9 & & \\
\hline University & 81 & 29.6 & & \\
\hline Other & 5 & 1.8 & & \\
\hline
\end{tabular}

TAFE: Technical and further education; PD: Parkinson's disease.

3.2. Multivariate Analyses. Four multiple linear regression models were constructed with forced entry of all univariate predictors (as determined above) in block 1, and personality variables entered in block 2 .

When controlling for the effects of patient disease duration, disease stage, caregiver age, depression, anxiety and hours assisting with ADLs for the physical health, and social relationship QoL domains, personality was no longer a significant predictor. The only significant predictor was depression $(b=-0.39, P<0.001)$ in the social relationship domain (full model $F_{7,223}=10.9, P<0.001$, adjusted $R^{2}=$ $23.1 \%)$.

Both neuroticism $(b=-0.14, t=-2.6, P<0.05)$ and conscientiousness $(b=0.10, t=2.0, P<0.05)$ 
TABLE 2: Univariate relationships between carer quality of life and personality.

\begin{tabular}{|c|c|c|c|c|}
\hline & \multicolumn{4}{|c|}{ Caregiver WHOQoL-BREF } \\
\hline & Physical health & Psychological health & Social relationship & Environment \\
\hline \multicolumn{5}{|l|}{ Patient } \\
\hline Disease duration & $-0.17^{* *}$ & $-0.17^{* *}$ & -0.11 & -0.12 \\
\hline Age & -0.10 & -0.02 & -0.02 & -0.04 \\
\hline Hoehn and Yahr [24] & $-0.18^{* *}$ & $-0.20^{* *}$ & $-0.18^{* *}$ & $-0.23^{* *}$ \\
\hline \multicolumn{5}{|l|}{ Caregiver } \\
\hline Age & $-0.15^{*}$ & 0.01 & -0.02 & -0.04 \\
\hline Duration of caregiving & $-0.32^{* *}$ & $-0.20^{* *}$ & $-0.21^{* *}$ & $-0.26^{* *}$ \\
\hline Hours per day assisting with ADL & $-0.22^{* *}$ & $-0.15^{*}$ & $-0.17^{* *}$ & $-0.34^{* *}$ \\
\hline Neuroticism & $-0.29^{* *}$ & $-0.44^{* *}$ & $-0.25^{* *}$ & $-0.28^{* *}$ \\
\hline Extroversion & 0.09 & $0.20^{* *}$ & 0.10 & 0.11 \\
\hline Agreeableness & -0.10 & 0.02 & 0.10 & -0.03 \\
\hline Conscientiousness & $0.17^{* *}$ & $0.21^{* *}$ & $0.14^{*}$ & 0.11 \\
\hline Openness & $0.13^{*}$ & 0.11 & 0.08 & $0.18^{* *}$ \\
\hline Anxiety & $-0.44^{* *}$ & $-0.63^{* *}$ & $-0.43^{* *}$ & $-0.57^{* *}$ \\
\hline Depression & $-0.53^{* *}$ & $-0.63^{* *}$ & $-0.47^{* *}$ & $-0.62^{* *}$ \\
\hline
\end{tabular}

remained significant predictors of psychological QoL even after controlling for the patient disease stage $(b=0.01, t=$ 0.1 , ns), disease duration ( $b=-0.11, t=-1.8, \mathrm{~ns})$, duration of caregiving $(b=0.01, t=0.2, \mathrm{~ns})$, number of hours spent assisting with $\operatorname{ADLs}(b=-0.02, t=-0.4, \mathrm{~ns})$, depression $(b=$ $-0.31, t=-3.9, P<0.001)$, and anxiety $(b=-0.31, t=-3.9$, $P<0.001)$. Overall, this combination of variables predicted $48.0 \%$ (adjusted $R^{2}$ ) of variance in psychological QoL (full model $\left.F_{9,221}=24.6, P<0.001\right)$. In contrast, extroversion was no longer a significant predictor after accounting for these other factors.

For the caregiver's environmental QoL, even after controlling for disease stage $(b=0.02, \mathrm{~ns})$, duration of caregiving $(b=-0.02, \mathrm{~ns})$, hours spent assisting with ADLs $(b=0.20$, $P<0.001)$, depression $(b=-0.40, P<0.001)$, and anxiety $(t=-0.19, P<0.001)$, the personality trait of openness remained a significant predictor $(b=0.13, P<0.01)$, though neuroticism did not. Overall, these variables explained $44.5 \%$ (adjusted $R^{2}$ ) of variance in environmental QoL (full model $\left.F_{7,223}=27.4, P<0.001\right)$.

\section{Discussion}

This is the first study to examine the relative contributions of personality to QoL in caregivers of PD patients. The main findings of this study confirm that depression and anxiety represent the largest negative predictors of QoL in caregivers and additionally highlight the role of personality factors. Neuroticism was negatively associated with psychological QoL amongst caregivers whereas conscientiousness, openness, and extroversion appeared to be positively protective. This large sample of PD patients and caregivers allowed multivariate modelling of personality attributes whilst controlling for possible confounds related to the patient and the caregiver. These analyses showed that personality variables did not predict QoL in social or physical domains, once all other confounds were considered.

Lower QoL in the psychological domain amongst caregivers was associated with being more neurotic. This suggests that caregivers who show a high level of neuroticism need increased levels of psychological support, as they may struggle to cope with stressful situations and an overbearing load of work. The psychological domain of QoL was associated with depression and anxiety emphasising the fact that these symptoms may be compounded in caregivers with increased rates of neuroticism. This highlights the need for an awareness of this combination of personality type and affective disorder. Such relationships have been highlighted in the spouse-caregivers of patients with Alzheimer's disease [25]. This work has identified a differential improvement in depression that was related to levels of neuroticism, suggesting that there is an opportunity to target psychological interventions for caregivers with lower levels of neuroticism [25]. A recent study evaluating a targeted intervention program for PD patients and their caregivers showed a beneficial effect on mood in both groups that lessened the psychosocial problems of the caregiver [26]. However, that study did not include a measurement of the caregiver's personality, which suggests that in clinical practice, incorporating screening of personality and psychological distress may be worthwhile to detect those caregivers for intervention programs early.

Better QoL in the psychological domain was associated with caregivers being more conscientious. Conscientious people have been shown to have problem-solving coping mechanism with strong attention regulation, which allows them to disengage from negative thoughts allowing them to have a better psychological health [11]. This coping method could be beneficial in caregivers with high levels of neuroticism $[16,27]$. A person who is conscientious may overcome unexpected obstacles more easily than a person who is less 
motivated to achieve important life tasks [12]. In this context, such people may gain a sense of pleasure and gratification from successfully operating in the role of a caregiver.

Poorer QoL in the environmental domain of the caregivers was associated with the hours they spent helping with ADLs, depression, and anxiety levels. This domain reflects issues relating to financial burden, concerns about physical safety, health and social care, and the home environment. Many of these concerns are very difficult to overcome but potentially could be improved by an increased knowledge and awareness about PD and the services available to support caregivers and patients in the rural or metropolitan areas [28]. It is hoped that such information could be successfully coordinated by having greater access to specialist PD nurses $[29,30]$. Interestingly, openness had a positive effect on the environmental domain of QoL amongst caregivers. This might reflect the fact that those caregivers having the openness personality trait tend to ask more questions and are amenable to new experiences, adapting to potential stressors.

One possible limitation of this study would be its reliance on self-reported questionnaires and it is feasible that clinical interview may have offered more detailed insights. However, these tools have all been well validated in previous work. Furthermore, the responses in a clinical interview might be more guarded by caregivers being concerned about judgment. Another limitation is that the briefer BFI-10 was used instead of the more in depth BFI-44, which may have lost some reliability, especially for the personality trait of agreeableness [20]. However, there were clear time constraints on the caregiver and a necessity to avoid overloading them. Future studies may seek to address this limitation by use of more indepth measures of personality. In terms of data interpretation, it is also possible that this study has an inflated type I error rate, since we did not apply a Bonferroni correction to the alpha levels used. Since this study was the first of its kind, we opted to leave the alpha level at 0.05 but acknowledge that future studies may wish to be more stringent in this regard.

Overall, this study supports that personality traits play a crucial role in the QoL of PD caregivers. Our results highlight that neuroticism negatively impacts on psychological wellbeing, even after controlling for other potential confounds. In contrast, psychological QoL was improved in those caregivers with higher levels of conscientiousness. A personality trait with more openness improves the QoL relating to the environment of the caregiver. It is hoped that a greater appreciation of these effects may help health professionals to better support these individuals and in turn support those patients living with PD.

\section{Authors' Contribution}

E. H. Tew integrated the literature, conducted preliminary data analysis, and took primary responsibility for initial drafting of the paper. M. Pereira carried out the assessments in the rural areas and assisted in the choice of questionnaires to implement for caregivers. S. J. G. Lewis and S. L. Naismith designed the study, provided guidance on the structuring of the paper, contributed to the content, revised several drafts of the paper, and conducted the multivariate analyses.

\section{Acknowledgments}

S. L. Naismith is supported by an NHMRC Career Development Award no. 1008117. S. J. G. Lewis is supported by an NHMRC Practitioner Fellowship no. 1003007 and a University of Sydney Rolf Edgar Lake Postdoctoral Fellowship. The authors would like thank all the participants who were involved in this research and the staff at the Parkinson's Disease Research Clinic at the BMRI.

\section{References}

[1] G. Alves, E. B. Forsaa, K. F. Pedersen, M. Dreetz Gjerstad, and J. P. Larsen, "Epidemiology of Parkinson's disease," Journal of Neurology, vol. 255, supplement 5, pp. 18-32, 2008.

[2] J. M. Glozman, "Quality of life of caregivers," Neuropsychology Review, vol. 14, no. 4, pp. 183-196, 2004.

[3] B. Thommessen, D. Aarsland, A. Braekhus, A. R. Oksengaard, K. Engedal, and K. Laake, "The psychosocial burden on spouses of the elderly with stroke, dementia and Parkinson's disease," International Journal of Geriatric Psychiatry, vol. 17, no. 1, pp. 78-84, 2002.

[4] J. H. Carter, B. J. Stewart, P. G. Archbold et al., "Living with a person who has Parkinson's disease: the spouse's perspective by stage of disease," Movement Disorders, vol. 13, no. 1, pp. 20-28, 1998.

[5] F. O’Reilly, F. Finnan, S. Allwright, G. D. Smith, and Y. BenShlomo, "The effects of caring for a spouse with Parkinson's disease on social, psychological and physical well-being," British Journal of General Practice, vol. 46, no. 410, pp. 507-512, 1996.

[6] S. H. Zarit, P. A. Todd, and J. M. Zarit, "Subjective burden of husbands and wives as caregivers: a longitudinal study," The Gerontologist, vol. 26, no. 3, pp. 260-266, 1986.

[7] P. Martínez-Martín, M. J. Forjaz, B. Frades-Payo et al., "Caregiver burden in Parkinson's disease," Movement Disorders, vol. 22, no. 7, pp. 924-931, 2007.

[8] A. Schrag, A. Hovris, D. Morley, N. Quinn, and M. Jahanshahi, "Caregiver-burden in Parkinson's disease is closely associated with psychiatric symptoms, falls, and disability," Parkinsonism and Related Disorders, vol. 12, no. 1, pp. 35-41, 2006.

[9] P. Martínez-Martín, S. Arroyo, J. M. Rojo-Abuin et al., "Burden, perceived health status, and mood among caregivers of Parkinson's disease patients," Movement Disorders, vol. 23, no. 12, pp. 1673-1680, 2008.

[10] L. R. Goldberg, "An alternative "description of personality": the big-five factor structure," Journal of Personality and Social Psychology, vol. 59, no. 6, pp. 1216-1229, 1990.

[11] C. S. Carver and J. Connor-Smith, "Personality and coping," Annual Review of Psychology, vol. 61, pp. 679-704, 2010.

[12] C. Wrosch and M. F. Scheier, "Personality and quality of life: the importance of optimism and goal adjustment," Quality of Life Research, vol. 12, supplement 1, pp. 59-72, 2003.

[13] J. M. Malouff, E. B. Thorsteinsson, and N. S. Schutte, "The relationship between the five-factor model of personality and symptoms of clinical disorders: a meta-analysis," Journal of Psychopathology and Behavioral Assessment, vol. 27, no. 2, pp. 101-114, 2005.

[14] P. Steel, J. Schmidt, and J. Shultz, "Refining the relationship between personality and subjective well-being," Psychological Bulletin, vol. 134, no. 1, pp. 138-161, 2008. 
[15] K. Hooker, D. J. Monahan, S. R. Bowman, L. D. Frazier, and K. Shifren, "Personality counts for a lot: predictors of mental and physical health of spouse caregivers in two disease groups," Journals of Gerontology B, vol. 53, no. 2, pp. 73-85, 1998.

[16] G. Melo, J. Maroco, and A. de Mendonça, "Influence of personality on caregiver's burden, depression and distress related to the BPSD," International Journal of Geriatric Psychiatry, vol. 26, no. 12, pp. 1275-1282, 2011.

[17] C. E. Löckenhoff, P. R. Duberstein, B. Friedman, and P. T. Costa, "Five-factor personality traits and subjective health among caregivers: the role of caregiver strain and self-efficacy," Psychology and Aging, vol. 26, no. 3, pp. 592-604, 2011.

[18] M. Abendroth, B. J. Lutz, and M. E. Young, "Family caregivers' decision process to institutionalize persons with Parkinson's disease: a grounded theory study," International Journal of Nursing Studies, vol. 49, no. 4, pp. 445-454, 2012.

[19] B. Goldsworthy and S. Knowles, "Caregiving for Parkinson's disease patients: an exploration of a stress-appraisal model for quality of life and burden," Journals of Gerontology B, vol. 63, no. 6, pp. P372-P376, 2008.

[20] B. Rammstedt and O. P. John, "Measuring personality in one minute or less: a 10-item short version of the big five inventory in English and German," Journal of Research in Personality, vol. 41, no. 1, pp. 203-212, 2007.

[21] "What quality of life? the WHOQOL group. World health organization quality of life assessment," World Health Forum, vol. 17, pp. 354-356, 1996.

[22] S. M. Skevington, M. Lotfy, and K. A. O'Connell, “The World health organization's WHOQOL-BREF quality of life assessment: psychometric properties and results of the international field trial. A report from the WHOQOL group," Quality of Life Research, vol. 13, no. 2, pp. 299-310, 2004.

[23] A. S. Zigmond and R. P. Snaith, "The hospital anxiety and depression scale," Acta Psychiatrica Scandinavica, vol. 67, no. 6, pp. 361-370, 1983.

[24] M. M. Hoehn and M. D. Yahr, "Parkinsonism: onset, progression and mortality," Neurology, vol. 17, no. 5, pp. 427-442, 1967.

[25] Y. Jang, O. J. Clay, D. L. Roth, W. E. Haley, and M. S. Mittelman, "Neuroticism and longitudinal change in caregiver depression: impact of a spouse-caregiver intervention program," The Gerontologist, vol. 44, no. 3, pp. 311-317, 2004.

[26] L. E. I. A'Campo, E. M. Wekking, N. G. A. Spliethoff-Kamminga, S. le Cessie, and R. A. C. Roos, "The benefits of a standardized patient education program for patients with Parkinson's disease and their caregivers," Parkinsonism and Related Disorders, vol. 16, no. 2, pp. 89-95, 2010.

[27] K. Hooker, L. D. Frazier, and D. J. Monahan, "Personality and coping among caregivers of spouses with dementia," The Gerontologist, vol. 34, no. 3, pp. 386-392, 1994.

[28] G. F. Duncan and P. Rositano, "Parkinson's disease in regional Australia," Rural and Remote Health, vol. 11, no. 4, article 1658, 2011.

[29] D. G. MacMahon, "Parkinson's disease nurse specialists: an important role in disease management," Neurology, vol. 52, no. 7, pp. S21-S25, 1999.

[30] B. Hurwitz, B. Jarman, A. Cook, and M. Bajekal, "Scientific evaluation of community-based Parkinson's disease nurse specialists on patient outcomes and health care costs," Journal of Evaluation in Clinical Practice, vol. 11, no. 2, pp. 97-110, 2005. 


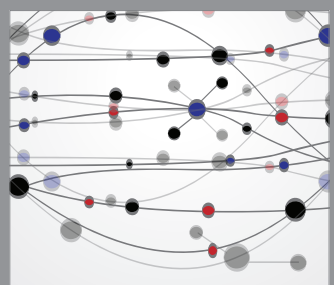

The Scientific World Journal
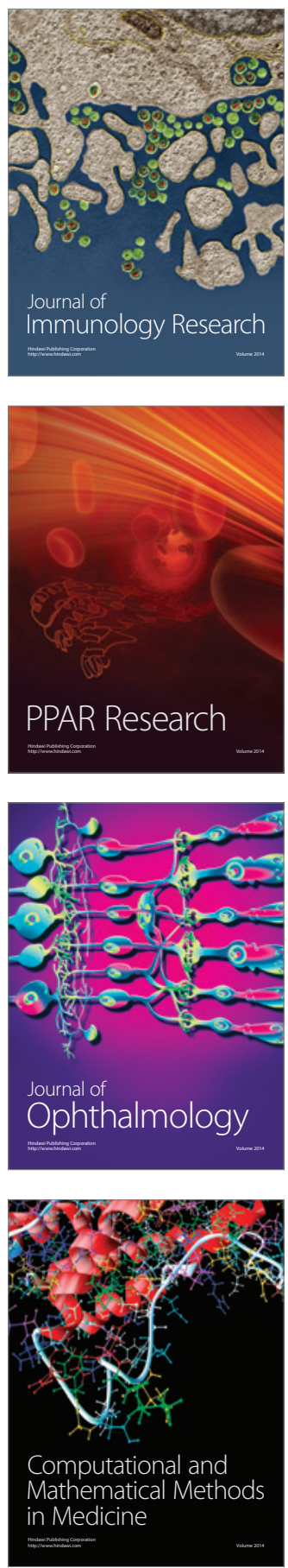

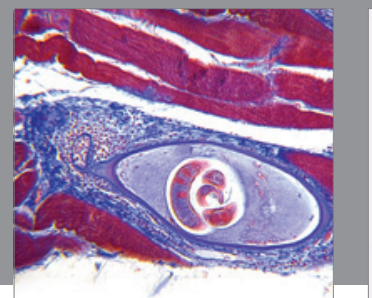

Gastroenterology

Research and Practice
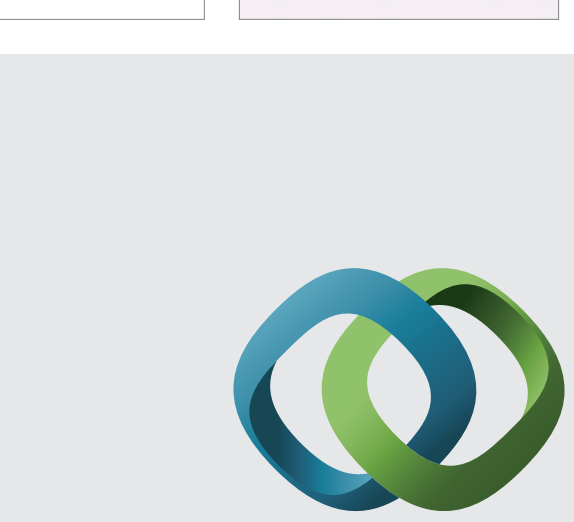

\section{Hindawi}

Submit your manuscripts at

http://www.hindawi.com
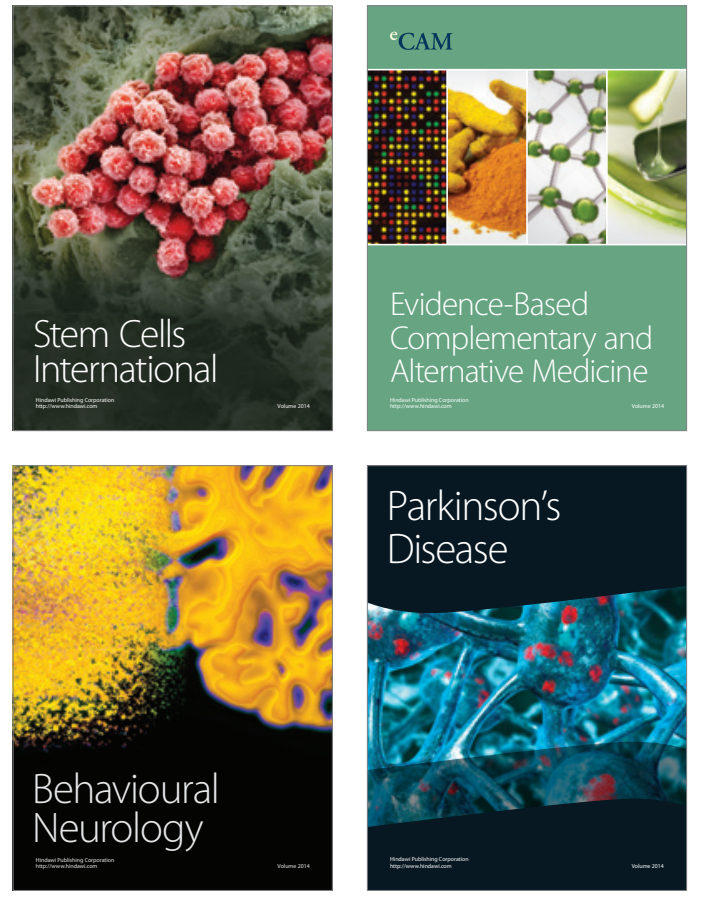
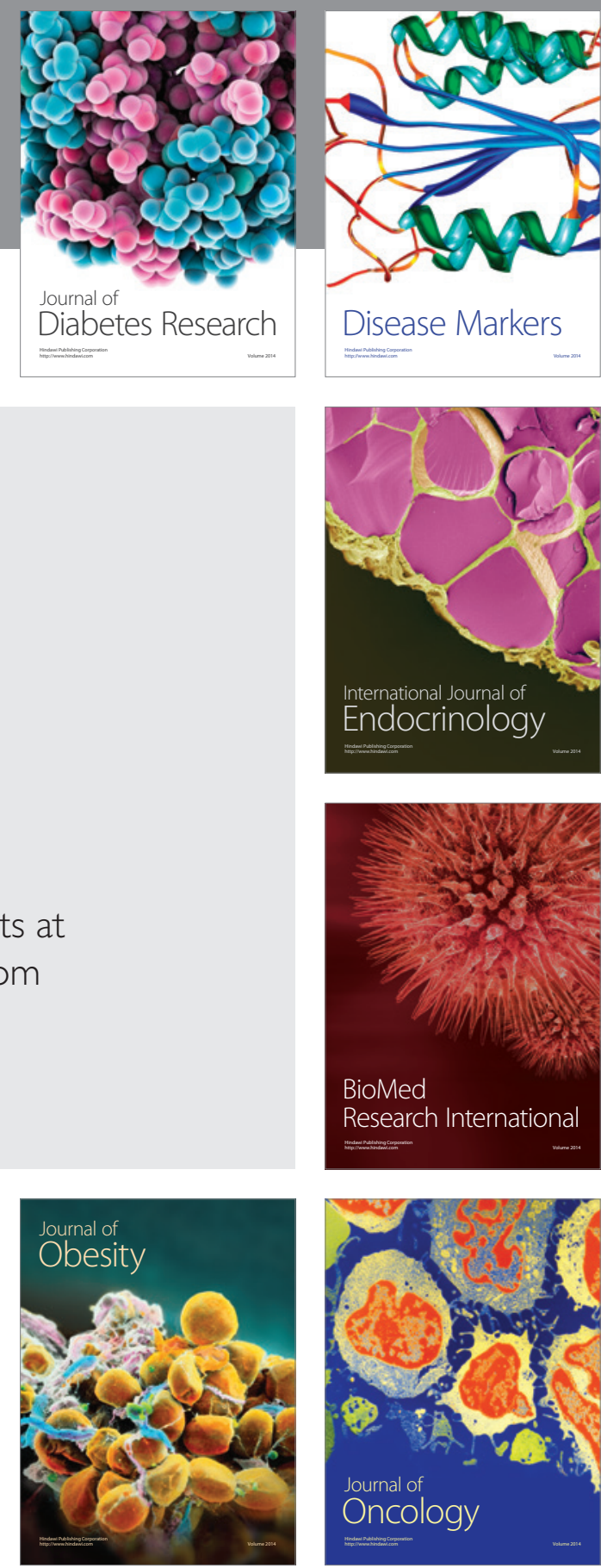

Disease Markers
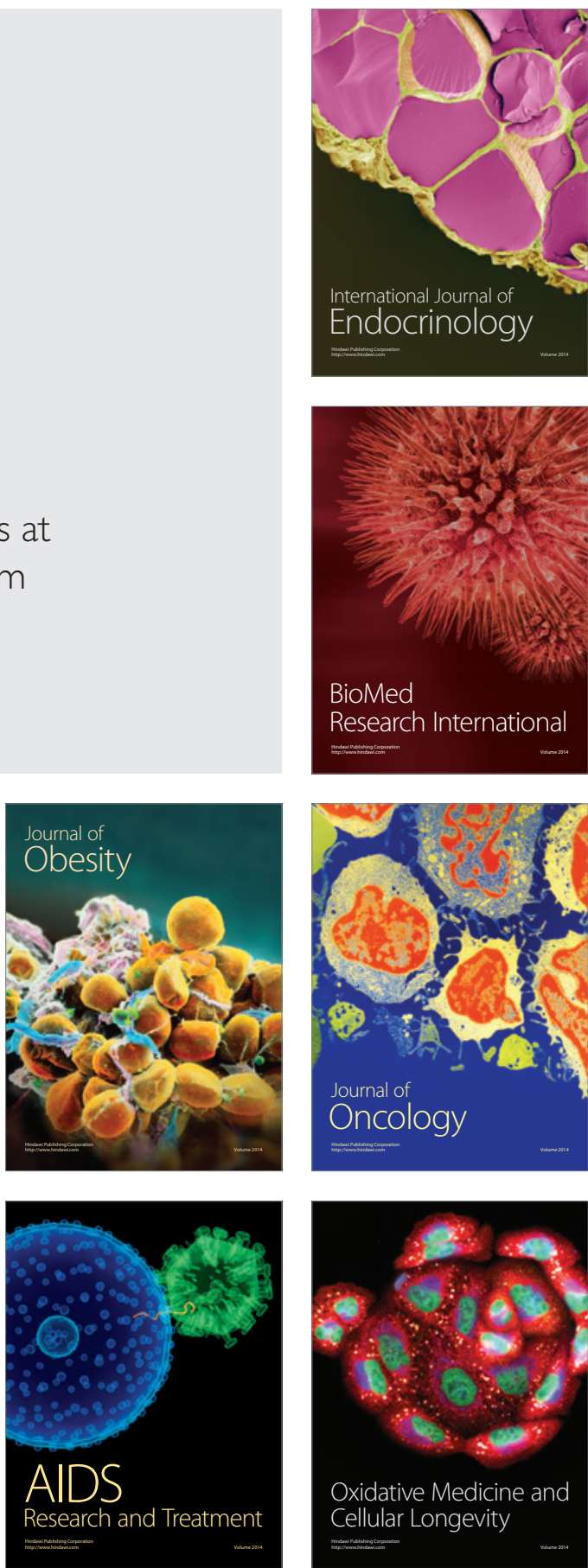